\title{
AN EXISTENCE THEOREM FOR SYSTEMS OF IMPLICIT DIFFERENTIAL EQUATIONS
}

\author{
THOMAS GRILL, MANFRED KNEBUSCH and MARCUS TRESSL \\ NWF-I Mathematik, Universität Regensburg, 93040 Regensburg, Germany \\ E-mail: manfred.knebusch@mathematik.uni-regensburg.de \\ marcus.tressl@mathematik.uni-regensburg.de
}

This note is based on the thesis [1] of the first author written under the guidance of the second author. The main technical input is Theorem 6 below. It will be proved in more generality in the subsequent paper [4].

Let $f_{1}, \ldots, f_{l}$ be differential polynomials in one derivative and $N$ variables with coefficients in $\mathbb{R}$. Suppose $I \subseteq \mathbb{R}$ is an open interval and $c: I \rightarrow \mathbb{R}^{N}$ is a $C^{\infty}$-map with $f_{1}(c(t))=\ldots=f_{l}(c(t))=0(t \in I)$. Let $\mathfrak{a}$ be the differential ideal generated by $f_{1}, \ldots, f_{l}$ in the differential polynomial ring $\mathbb{R}\left\{X_{1}, \ldots, X_{N}\right\}$. Then $\mathfrak{a}$ is certainly a semireal ideal, i.e. for all $g_{1}, \ldots, g_{m} \in \mathbb{R}\left\{X_{1}, \ldots, X_{N}\right\}$ we have $1+\sum_{j=1}^{m} g_{j}^{2} \notin \mathfrak{a}$. This follows immediately from our assumption that $c$ is a differential solution of the generators $f_{1}, \ldots, f_{l}$ of a. We'll prove here the converse of this observation, in other words we'll prove

THEOREM 1. If $\mathfrak{a}$ is a differential ideal of $\mathbb{R}\left\{X_{1}, \ldots, X_{N}\right\}$ and $\mathfrak{a}$ is semireal, then there is some nonempty open interval $I \subseteq \mathbb{R}$ and an analytic map $c: I \rightarrow \mathbb{R}^{N}$ with $f(c(t))=0$ $(f \in \mathfrak{a}, t \in I)$.

In order to find an analytic map $c=\left(c_{1}, \ldots, c_{N}\right): I \rightarrow \mathbb{R}^{N}$ solving each relation $f=0$ with $f \in \mathfrak{a}$ it is enough to find a nonempty open interval $I$ of $\mathbb{R}$ together with a differential homomorphism $\mathbb{R}\left\{X_{1}, \ldots, X_{N}\right\} / \mathfrak{a} \rightarrow C^{\omega}(I)$ and then take $c_{i}:=$ the image of $X_{i} \bmod \mathfrak{a}$ under this map. We divide this problem into an algebraic part (Theorem 2) and an analytic part (Proposition 3).

TheOREM 2. Let $F$ be a differential field and let $A$ be a differentially finitely generated $F$-algebra. Suppose $A$ is semireal, i.e. -1 is not a sum of squares in $A$. There is a real, differential $F$-algebra $C$, which is an integral domain and finitely generated as an $F$ algebra together with a differential F-algebra homomorphism $A \rightarrow C$.

2000 Mathematics Subject Classification: Primary 12H05; Secondary 12D.

The paper is in final form and no version of it will be published elsewhere. 
Proposition 3. Let $C$ be a real, differential $\mathbb{R}$-algebra, which is an integral domain and finitely generated as an $\mathbb{R}$-algebra. Then there is a differential $\mathbb{R}$-algebra homomorphism $C \rightarrow C^{\omega}(I)$ for some open interval $I \subseteq \mathbb{R}$.

Clearly 1 follows from 2 and 3 applied to $A=\mathbb{R}\left\{X_{1}, \ldots, X_{N}\right\} / \mathfrak{a}$. Before we prove Theorem 2 and Proposition 3 we need some real algebraic preparations.

Definition 1. A ring $A$ is called semireal if -1 is not a sum of squares in $A$. $A$ is called real if $a_{1}^{2}+\ldots+a_{n}^{2}=0$ implies $a_{1}=\ldots=a_{n}=0$ for all $n \in \mathbb{N}$ and all $a_{1}, \ldots, a_{n} \in A$. An ideal $\mathfrak{a}$ of $A$ is called (semi) real if the $\operatorname{ring} A / \mathfrak{a}$ is (semi) real.

Definition 2. Let $A$ be a differential ring in $K$ derivatives and let $\mathfrak{a}$ be an ideal of $A$. We define

$$
\mathfrak{a}^{\#}:=\{a \in \mathfrak{a} \mid \text { every derivative of } a \text { is in } \mathfrak{a}\} .
$$

The useful construction $\mathfrak{a}^{\#}$ was first introduced by Keigher in [2]. Clearly $\mathfrak{a}^{\#}$ is the largest differential ideal of $A$ contained in $\mathfrak{a}$. Let $\sigma: A \rightarrow B$ a ring homomorphism into a ring $B$. Let $B[[T]]$ be the power series ring over $B$ in one variable $T . B[[T]]$ is a differential ring with the standard derivative $\frac{d}{d T}$. We define the Taylor morphism $T_{\sigma}: A \rightarrow B[[T]]$ by

$$
T_{\sigma}(a):=\sum_{n \geq 0} \frac{\sigma\left(d^{n} a\right)}{n !} T^{n} .
$$

Here $d^{n} a$ denotes the $n$-th derivative of $a \in A$.

The Leibniz rule implies that $T_{\sigma}$ is a differential homomorphism. If $\sigma: A \rightarrow A / \mathfrak{a}$ is the residue map corresponding to an ideal $\mathfrak{a}$ of $A$, then clearly $\mathfrak{a}^{\#}$ is the kernel of $T_{\sigma}$.

Proposition 4. Let $\mathfrak{a}$ be an ideal in the differential ring A. If $\mathfrak{a}$ is prime, semireal, real respectively, then $\mathfrak{a}^{\#}$ is prime, semireal, real respectively.

Proof. If $\mathfrak{a}$ is prime, semireal, real respectively, then $A / \mathfrak{a}$ is a domain, semireal, real respectively. Hence the power series ring $A / \mathfrak{a}[[T]]$ is a domain, semireal, real respectively, and so $\mathfrak{a}^{\#}=\operatorname{Ker}\left(T_{A \rightarrow A / \mathfrak{a}}\right)$ is prime, semireal, real respectively.

Proposition 5. Let $A$ be a differential ring and let $\mathfrak{p} \subseteq A$ be a differential ideal. Then $\mathfrak{p}$ is maximal among the proper, semireal and differential ideals of $A$ if and only if $\mathfrak{p}$ is maximal among the proper, real and differential ideals of $A$. In this case $\mathfrak{p}$ is prime.

Proof. Let $\mathfrak{p}$ be maximal among all proper, semireal and differential ideals of $A$. The Proposition is proved if we can show that $\mathfrak{p}$ is real and prime. By classical real algebra (cf. [3], III, §3, Satz 2), there is a real prime ideal $\mathfrak{q}$ of $A$ containing $\mathfrak{p}$. By Proposition $4, \mathfrak{q}^{\#}$ is a real, differential prime ideal of $A$. Since $\mathfrak{q}^{\#}$ contains $\mathfrak{p}$, the maximality of $\mathfrak{p}$ implies $\mathfrak{p}=\mathfrak{q}^{\#}$, thus $\mathfrak{p}$ is real and prime.

Finally we use a structure theorem for differential algebras (in one derivative), as explained in [4].

THEOREM 6. Let $S=(S, d)$ be a differential domain in one derivative, containing $\mathbb{Z}$, and let $R=(R, d) \subseteq(S, d)$ be a differential subring such that $S$ is differentially finitely generated over $R$. Then there are $R$-subalgebras $B$ and $U$ of $S$ and an element $h \in B$, $h \neq 0$ such that: 
(a) $B$ is a finitely generated $R$-algebra and $B_{h}$ is a finitely presented $R$-algebra.

(b) $S_{h}=(B \cdot U)_{h}$ is a differentially finitely presented $R$-algebra.

(c) The homomorphism $B \otimes_{R} U \rightarrow B \cdot U$ induced by multiplication is an isomorphism of $R$-algebras.

(d) $U$ is a differential polynomial ring over $R$ in finitely many variables.

Proof. This is Theorem 1 in [4] for the case of one derivative. Take $U:=P_{\{d\}}$ and replace $B$ by $B \cdot P_{\emptyset}$ in [4], Theorem 1.

Proof of Theorem 2. Since $A$ is semireal, $A$ contains an ideal $\mathfrak{p}$, which is maximal among all proper, semireal and differential ideals of $A$. By Proposition $5, \mathfrak{p}$ is a real, differential prime ideal. Let $S$ be the differential $F$-algebra $S:=A / \mathfrak{p}$. Take $F$-subalgebras $B, U$ of $S$ and an element $h \in B, h \neq 0$ as in Theorem 6. Since $S$ is real, $B$ and $B_{h}$ are real, too. It is enough to show that $U=F$, then the differential map $A \rightarrow A / \mathfrak{p}=$ $S \hookrightarrow S_{h}=B_{h}=: C$ has the required properties. Suppose $U \neq F$. Since $B_{h}$ is a finitely generated, real $F$-algebra, Tarski's principle gives a homomorphism $\varphi: B_{h} \rightarrow \bar{F}$ into a real closed field $\bar{F}$ containing $F$. Since $U \neq F$ is a differential polynomial ring, there is a differential $F$-algebra homomorphism $\tau: U \rightarrow F$ with nontrivial kernel. By Theorem 6 , there is an $F$-algebra homomorphism $\sigma: S \rightarrow \bar{F}$, extending $\left.\varphi\right|_{B}$ and $\tau$. Thus $\mathfrak{q}:=\operatorname{Ker} \sigma$ is a real ideal of $S$ containing $\operatorname{Ker} \tau$. By Proposition $4, \mathfrak{q}^{\#}$ is a real, differential ideal of $S$. Since $\tau$ is a differential homomorphism, $\mathfrak{q}^{\#}$ contains $\operatorname{Ker} \tau$, hence $\mathfrak{q}^{\#}$ is a nontrivial, real, differential ideal of $S$, which contradicts the maximality of $\mathfrak{p}$.

Proof of Proposition 3. Let $C=\mathbb{R}\left[a_{1}, \ldots, a_{n}\right]$ and let $g_{i} \in \mathbb{R}\left[X_{1}, \ldots, X_{n}\right]$ such that $g_{i}(a)$ is the derivative of $a_{i}$ in $C$. We consider the $\operatorname{ring} \mathbb{R}\left[X_{1}, \ldots, X_{n}\right]$ as a differential ring with derivation $d: \mathbb{R}\left[X_{1}, \ldots, X_{n}\right] \rightarrow \mathbb{R}\left[X_{1}, \ldots, X_{n}\right]$ defined by $d X_{i}=g_{i}$. Then the homomorphism $\lambda: \mathbb{R}\left[X_{1}, \ldots, X_{n}\right] \rightarrow C$ sending $X_{i}$ to $a_{i}$ is differential. Since $C$ is a real, finitely generated $\mathbb{R}$-algebra, there is an $\mathbb{R}$-algebra homomorphism $\varepsilon: C \rightarrow \mathbb{R}$. The fundamental theorem on ordinary differential equations gives an open interval $I$ of $\mathbb{R}$ containing 0 and analytic maps $c_{i}: I \rightarrow \mathbb{R}(1 \leq i \leq n)$ such that $c_{i}(0)=\varepsilon\left(a_{i}\right)$ and

$$
c_{i}^{\prime}(t)=g_{i}\left(c_{1}(t), \ldots, c_{n}(t)\right)(1 \leq i \leq n) .
$$

Now a straightforward computation shows that the Taylor morphism $T_{\varepsilon}$ of $\varepsilon: C \rightarrow \mathbb{R}$ maps $a_{i}$ to the Taylor expansion of $c_{i}$ at 0 . By shrinking $I$ if necessary, we get that $T_{\varepsilon}$ has values in $C^{\omega}(I)$, which proves Proposition 3 .

\section{References}

[1] T. Grill, Contributions to differential, real algebra and its connection to differential equations, Dissertation, Regensburg, 1997.

[2] W. F. Keigher, Prime differential ideals in differential rings, in: Contributions to Algebra, H. Bass, P. J. Cassidy and J. Kovacic (eds.), Academic Press, New York, 1977, 239-249.

[3] M. Knebusch and C. Scheiderer, Einführung in die reelle Algebra, Vieweg, 1989.

[4] M. Tressl, A structure theorem for differential algebras, this volume. 